\title{
Spatially resolved molecular gas in early-type galaxies
}

\author{
T. A. Davis, ${ }^{1}$ K. Alatalo, ${ }^{2}$ M. Bureau, ${ }^{3}$ L. Young, ${ }^{4}$ L. Blitz, ${ }^{2}$ \\ A. Crocker ${ }^{5}$ E. Bayet, ${ }^{3}$ M. Bois, ${ }^{6}$ F. Bournaud, ${ }^{7}$ M. Cappellari,${ }^{3}$ \\ R. L. Davies, ${ }^{3}$ P-A. Duc, ${ }^{7}$ P. T. de Zeeuw, ${ }^{1,8}$ E. Emsellem, ${ }^{1,9}$ \\ J. Falcon-Barroso, ${ }^{10}$ S. Khochfar, ${ }^{11}$ D. Krajnovic, ${ }^{1}$ H. Kuntschner, ${ }^{1}$ \\ P.-Y. Lablanche, ${ }^{1}$ R. M. McDermid, ${ }^{12}$ R. Morganti, ${ }^{13}$ T. Naab, ${ }^{14}$ \\ M. Sarzi, ${ }^{15}$ N. Scott,${ }^{16}$ P. Serra, ${ }^{13}$ and A. Weijmans ${ }^{17}$ \\ ${ }^{1}$ ESO, Germany; ${ }^{2}$ UC Berkeley, USA $;{ }^{3}$ Univ. Oxford, UK; ${ }^{4}$ New Mexico Tech, USA $;{ }^{5}$ Univ. \\ Toledo, USA; ${ }^{6}$ Observatoire de Paris, France; ${ }^{7}$ Univ. Paris Diderot, France; ${ }^{8}$ Leiden Univ., \\ The Netherlands; ${ }^{9}$ Univ. de Lyon, France; ${ }^{10}$ IAC, Spain; ${ }^{11}$ MPE, Germany; ${ }^{12}$ Gemini \\ Observatory, USA; ${ }^{13}$ ASTRON, The Netherlands; ${ }^{14} \mathrm{MPA}$, Germany; ${ }^{15}$ Univ. Hertfordshire, \\ UK; ${ }^{16}$ Swinburne Univ., Australia; ${ }^{17}$ Univ. Toronto, Canada;
}

\begin{abstract}
In around $\approx 25 \%$ of early-type galaxies (ETGs) UV emission from young stellar populations is present. Molecular gas reservoirs have been detected in these systems (e.g. Young et al. 2011), providing the fuel for this residual star-formation. The environment in which this molecular gas is found is quite different than that in spiral galaxies however, with harsher radiation fields, deeper potentials and high metallicity and alpha-element abundances. Here we report on one element of our multi-faceted programme to understand the similarities and differences between the gas reservoirs in spirals and ETGs. We use spatially resolved observations from the CARMA mm-wave interferometer to investigate the size of the molecular reservoirs in the the CO-rich ATLAS ${ }^{3 \mathrm{D}}$ ETGs (survey described in Alatalo et al. 2012, submitted). We find that the molecular gas extent is smaller in absolute terms in ETGs than in late-type galaxies, but that the size distributions are similar once scaled by the galaxies optical/stellar characteristic scale-lengths (Fig 1, left). Amongst ETGs, we find that the extent of the molecular gas is independent of the kinematic misalignment, despite the many reasons why misaligned gas might have a smaller extent. The extent of the molecular gas does depend on environment, with Virgo cluster ETGs having less extended molecular gas reservoirs (Fig 1, right). Whatever the cause, this further emphases that cluster ETGs follow different evolutionary pathways from those in the field. Full details of this work will be presented in Davis et al. (2012), submitted.
\end{abstract}

\section{Reference}

Young, L. M., Bureau, M., Davis, T. A., et al. 2011, MNRAS, 414, 940 


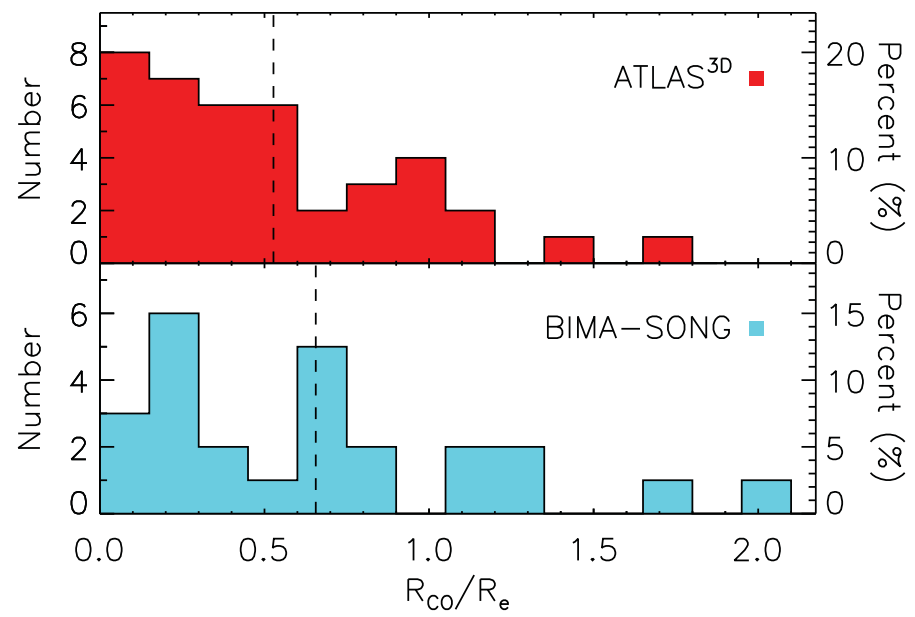

Figure 1. Molecular sizes for the ATLAS ${ }^{3 \mathrm{D}}$ ETGs normalised by the stellar effective radius. Compared to BIMA-SONG spirals (left) and as a function of environment (right). The mean radius is marked with a dashed line. 